\title{
Diacronie
}

Studi di Storia Contemporanea

$N^{\circ} 32,4 \mid 2017$

Proiezioni individuali e agire collettivo nella storia

\section{Mirco Dondi, L'eco del boato. Storia della strategia della tensione 1965-1974}

\section{Luca Bufarale}

\section{Q OpenEdition}

Edizione digitale

URL: http://journals.openedition.org/diacronie/6939

DOI: 10.4000/diacronie.6939

ISSN: 2038-0925

Editore

Association culturelle Diacronie

Notizia bibliografica digitale

Luca Bufarale, «Mirco Dondi, L'eco del boato. Storia della strategia della tensione 1965-1974», Diacronie [Online], N 32, 4 | 2017, documento 12, Messo online il 29 décembre 2017, consultato il 24 septembre 2020. URL : http://journals.openedition.org/diacronie/6939; DOI : https://doi.org/10.4000/diacronie. 6939 


\section{Diacronie}

Studi di Storia Contemporanea

\section{$32,4 / 2017$}

Proiezioni individuali e agire collettivo nella storia. Ruoli sociali, aspetti politici e nodi storiografici tra pubblico e privato.

\section{RECENSIONE: Mirco DONDI, L'eco del boato. Storia della strategia della tensione 1965-1974, Roma-Bari, Laterza, 2015, 445 pp.}

Per citare questo articolo:

BUFARALE, Luca, «RECENSIONE: Mirco DONDI, L'eco del boato. Storia della strategia della tensione 1965-1974, Roma-Bari, Laterza, 2015, 445 pp.», Diacronie. Studi di Storia Contemporanea : Proiezioni individuali e agire collettivo nella storia. Ruoli sociali, aspetti politici e nodi storiografici tra pubblico e privato, 32, 4/2017, 29/12/2017.

URL: < http://www.studistorici.com/2017/12/29/bufarale_numero_32/>

Diacronie Studi di Storia Contemporanea $\rightarrow$ http://www.diacronie.it Rivista storica online. Uscita trimestrale.

redazione.diacronie@hotmail.it

Comitato di direzione: Naor Ben-Yehoyada - João Fábio Bertonha - Christopher Denis-Delacour - Maximiliano Fuentes Codera Anders Granås Kjøstvedt - John Paul Newman - Deborah Paci - Niccolò Pianciola - Spyridon Ploumidis - Wilko Graf Von Hardenberg

Comitato di redazione: Jacopo Bassi - Luca Bufarale - Gianluca Canè - Fausto Pietrancosta - Alessandro Salvador - Matteo Tomasoni - Luca Zuccolo 


\section{2/ RECENSIONE: Mirco DONDI, L'eco del boato. Storia della strategia della tensione 1965-1974, Roma-Bari, Laterza, 2015, 445 pp.}

Io so.

Io so i nomi dei responsabili di quello che viene chiamato golpe (e che in realtà è una serie di golpes istituitasi a sistema di protezione del potere) $)^{1}$.

Più di quarant'anni sono passati dal celebre articolo di Pier Paolo Pasolini che sul «Corriere della Sera» denunciava la mano di esponenti del governo, di settori delle forze armate e di polizia e dei servizi segreti italiani e statunitensi dietro le stragi compiute da gruppi neofascisti, da Piazza Fontana a Milano (12 dicembre 1969) sino alla bomba del treno Italicus sulla linea Bologna-Firenze (4 agosto 1974).

Io so - aggiungeva Pasolini - ma non ho le prove. Non ho nemmeno indizi. Io so perché sono un intellettuale, uno scrittore, che cerca di seguire tutto quello che succede, di conoscere tutto ciò che se ne scrive, di immaginare tutto ciò che non si sa o che si tace; che coordina fatti anche lontani, che mette insieme i pezzi disorganizzati e frammentari di un intero coerente quadro politico, che ristabilisce la logica là dove sembrano regnare l'arbitrarietà, la follia e il mistero ${ }^{2}$.

A quattro decenni di distanza, dopo travagliati iter giudiziari, perlomeno alcune tessere del mosaico cominciano a comporre un quadro coerente. Eppure la strategia della tensione resta una

Desidero ringraziare Luca Pastore dell'Istituto storico della Resistenza di Bologna per lo scambio di idee sulla strategia della tensione e gli utili consigli bibliografici.

${ }^{1}$ PASOLINI, Pier Paolo, «Che cos'è questo golpe?», in Corriere della Sera, 14 novembre 1974, ora in ID., Scritti corsari, Milano, Garzanti, 2008 [Ed. orig. 1975], p. 88.

${ }^{2}$ Ibidem, p. 89. Sul rapporto tra lo scrittore e la strategia della tensione cfr. BOLOGNESI, Paolo, SPERANZONI, Andrea, Pasolini. Un omicidio politico, Roma, Castelvecchi, 2017. 
questione aperta, soprattutto a livello storiografico. Quali sono stati i suoi attori principali e come si muovevano? Quali i fini che si prospettavano? Che impatto ha avuto la strategia della tensione nella società italiana e come è stata recepita all'estero? Sono ancora pochi i saggi che provano a dare una risposta a tali questioni, da un punto di vista che non sia solo quello dell'inchiesta giornalistica o dello studio su un singolo caso.

Le esitazioni degli storici ad occuparsi di una materia che può apparire sin troppo incandescente sono comprensibili e il rischio di basare la propria ricostruzione su ipotesi non verificate è reale. Non stupisce che negli anni Novanta l'uscita dei saggi di Franco De Felice e Nicola Tranfaglia sulla tematica del "doppio stato" sia stata accompagnata da vivaci polemiche ${ }^{3}$. Ma questo vuoto storiografico finisce per avere pesanti conseguenze nella rappresentazione del decennio che va dalla fine degli anni Sessanta alla fine dei Settanta. Diventa dominante un ritratto della lotta politica di quel periodo che vede contrapposti in modo troppo semplicistico "rossi" e "neri", in una sorta di riproposizione della teoria - più ideologico-politica che storiografica - degli opposti estremismi. Una raffigurazione che si ritrova di frequente anche in buona parte della memorialistica, nella manualistica scolastica, nel cinema. Basti pensare, per quest'ultimo aspetto, al successo di film come La meglio gioventù o Mio fratello è figlio unico (ma si potrebbero citare anche altri esempi) dove il tema della strategia della tensione è praticamente assente e il decennio 1967-77 appare dominato soprattutto dallo scontro tra estremisti di destra e di sinistra e dalla deriva violenta di questi ultimi, seguita ad un Sessantotto caratterizzato da impegno civile e lotte dure ma ancora sostanzialmente pacifiche. Tanto che, sempre rimanendo in ambito cinematografico, per avere un'idea della strategia della tensione e dei suoi corollari - colpi di stato reali o progettati, trame occulte, depistaggi, mass media compiacenti - bisogna ricorrere (salvo qualche eccezione come il recente discusso film Romanzo di una strage ${ }^{5}$ ) soprattutto a pellicole prodotte in quegli stessi anni, come Indagine su un cittadino al di sopra di ogni sospetto, Sbatti il mostro in prima pagina o Vogliamo i colonnelli ${ }^{6}$.

Mirco Dondi si prefigge di colmare questa lacuna. Non è la prima volta che l'autore - che insegna storia contemporanea a Bologna e dirige presso lo stesso ateneo il Master di Comunicazione storica - affronta con passione civile e rigore storiografico temi "scomodi". Lo aveva già fatto, ad esempio, con La lunga liberazione, dedicato alla difficile transizione post-25

\footnotetext{
${ }^{3}$ Il riferimento è a DE FELICE, Franco, Doppia lealtà e doppio Stato, in «Studi storici», 3/1989, pp. 493-563; TRANFAGLIA, Nicola, Un capitolo del "doppio stato". La stagione delle stragi e dei terrorismi (1969-1984), in Storia dell'Italia repubblicana, III, vol. $2^{\circ}$, Torino Einaudi, 1997, pp. 7-80. Per una ricostruzione del dibattito riguardo alle tesi sul "doppio Stato" cfr. CECI, Giovanni Mario, Il terrorismo italiano. Storia di un dibattito, Roma, Carocci, 2013, pp. 204-216.

${ }^{4}$ GIORDANA, Marco Tullio, La meglio gioventù, Miramax Films, Italia 2003, 360'; LUCHETTI, Daniele, Mio fratello è figlio unico, Cattleya, Italia 2007, 100'.

${ }^{5}$ GIORDANA, Marco Tullio, Romanzo di una strage, Cattleya, Italia-Francia 2012, 129'.

${ }^{6}$ PETRI, Elio, Indagine su un cittadino al di sopra di ogni sospetto, Vera Film, Italia 1970, 112'; BELLOCCHIO, Marco, Sbatti il mostro in prima pagina, Jupiter Generale Cinematografica-UTI Produzioni Associate-Labrador Films, Italia 1972, 83'; MONICELLI, Mario, Vogliamo i colonnelli, Dean Film, Italia 1973, 100'.
} 
aprile $^{7}$. L'autore vuole offrire una visione d'insieme dello stragismo che insanguinò l'Italia dal 1969 al 1974, dei rapporti che settori dei servizi segreti italiano e statunitense e personalità della politica, delle forze armate e di polizia intrattenevano con organizzazioni eversive di estrema destra, delle strategie messe in campo per il depistaggio delle stragi e, infine, dell'impatto dello stragismo nella magistratura, nelle forze politiche e più in generale presso l'opinione pubblica.

Quest'ultimo aspetto risulta decisivo e spiega anche il titolo del volume. L'eco del boato, infatti, indica «le forme di condizionamento» che i mass media mettono in campo per indirizzare il pubblico verso una certa pista, utilizzando la notizia di un attentato per attaccare un certo avversario politico e creare un determinato clima nella società ${ }^{8}$. Decisiva, in questo senso, si rivela, assai più che la televisione - siamo ancora in un'epoca paleotelevisiva, anteriore cioè alla spettacolarizzazione degli eventi attraverso la TV che in Italia si avrà soprattutto dagli anni Ottanta - la stampa. Giornali, riviste e opuscoli non vengono utilizzati da Dondi solo come fonte (accanto alle fonti giudiziarie e alla memorialistica), ma costituiscono l'oggetto privilegiato di indagine proprio perché hanno contribuito in maniera decisiva a creare la strategia della tensione, o, a seconda dei casi, a combatterla.

Ufficialmente, si inizia a parlare di strategia della tensione solo nel 1969, all'indomani dell'eccidio di Piazza Fontana, in seguito ad un articolo del settimanale britannico «The Observer» che conia questa espressione mettendone insieme altre due: la strategia dell'attenzione, con riferimento alle aperture del leader democristiano Aldo Moro rispetto al Partito comunista (che, anche in seguito alla condanna dell'intervento sovietico in Cecoslovacchia, si sta distanziando dall'URSS), e la politica della distensione tra Stati Uniti e Unione Sovietica. La strategia della tensione indica così una precisa politica, messa in atto da determinati settori delle istituzioni, per strumentalizzare gli episodi di violenza e condizionare il quadro politico e sociale favorendo una svolta in senso contrario ad un'apertura alle forze di sinistra. Tutto ciò in un contesto che, in seguito alle lotte studentesche del 1967-68 e all'“autunno caldo" del 1969, appare in rapida evoluzione, dopo la stagione del centrismo degli anni Cinquanta e quella del centro-sinistra degli anni Sessanta. La stampa di orientamento reazionario, moderato o comunque filogovernativo e le stesse agenzie di informazioni si rivelano fondamentali per la copertura mediatica degli eventi e per orientare l'opinione pubblica. Così, ad esempio, l'attribuzione dell'attentato di Piazza Fontana al gruppo anarchico di Valpreda e Pinelli si rivela un meccanismo costruito ad arte e poi amplificato dai mass media. Le conseguenze (ma per alcuni si tratta di fini coscientemente perseguiti) sono: diffondere un clima di paura e incertezza e criminalizzare l'estrema sinistra legittimando un'evoluzione in senso più autoritario dello stato.

L'autore, tuttavia, sceglie il 1965 come termine a quo della strategia della tensione: nel maggio di quell'anno, infatti, si svolge a Roma un convegno dell'Istituto Pollio finanziato indirettamente

\footnotetext{
${ }^{7}$ DONDI, Mirco, La lunga liberazione. Giustizia e violenza nel dopoguerra italiano, Roma, Editori Riuniti, 1999.

${ }^{8}$ DONDI, Mirco, L'eco del boato. Storia della strategia della tensione 1965-1974, Roma-Bari, Laterza, 2015, p. 3.
} 
dal SIFAR (servizio segreto militare, poi sciolto e rinato come SID) a cui partecipano, oltre che esponenti delle forze armate, dei servizi segreti e del mondo politico (dall'estrema destra di Pino Rauti e Stefano Delle Chiaie a un dirigente socialdemocratico come Ivan Matteo Lombardo), anche molti giornalisti, tra cui i direttori dei quotidiani «Il Messaggero», «Il Tempo» e «La Nazione» e di settimanali come «Lo Specchio» e «Il Borghese» ${ }^{9}$. Dal convegno nascono i Nuclei di difesa dello Stato, strumento per la guerra non ortodossa, esplicitamente finalizzata alla repressione interna. L'obiettivo è «destabilizzare per stabilizzare»: si tratta di ricorrere ad azioni terroristiche da attribuire poi all'avversario. Solo in questo modo i promotori ritengono che sia possibile combattere le sinistre, giustificare di fronte all'opinione pubblica una stretta repressiva e addirittura un golpe più o meno mascherato. Sciolti nel 1973, i Nuclei di difesa dello Stato verranno scoperti soltanto venti anni più tardi dalle indagini dei giudici Leonardo Grassi e Guido Salvini.

Il primo capitolo del libro fornisce una ricostruzione del contesto in cui matura la strategia della tensione e dei suoi attori principali all'interno delle istituzioni, nei suoi rapporti con l'intelligence statunitense e nelle formazioni di estrema destra come Avanguardia Nazionale ed Ordine Nuovo. Il secondo capitolo si sofferma invece sul ruolo di giornali e agenzie di stampa. Il peso delle agenzie nell'orientare l'opinione pubblica è spesso stato trascurato. Eppure - nota giustamente Dondi - «la prima trasformazione del fatto accaduto in notizia è opera delle agenzie che con la selezione delle notizie e i primi commenti tendono a influenzare la stampa ${ }^{10}$. Alcune (D, Oltremare) sono fondate da membri di Ordine Nuovo, altre come Op o Aipe hanno legami con i servizi segreti. A volte la competizione tra questi ultimi è alla base della diffusione di informazioni riservate: se nel 1967, ad esempio, è l'Ufficio affari riservati (UAARR, dipendente dal Ministero dell'Interno) a fornire al settimanale «L'Espresso» le informazioni alla base dello scoop sul Piano Solo (che tre anni prima aveva conseguito il risultato di "moderare" le pretese dell'appena costituito governo di centro-sinistra) con l'intento di delegittimare il servizio segreto militare (SID), nel 1970 sarà quest'ultimo, in contrasto con l'UAARR, a divulgare le prime rivelazione sulla "pista nera" della strage di Piazza Fontana ${ }^{11}$.

Il terzo capitolo ricostruisce alcuni atti di violenza che nel 1969 precedono questa strage. Tra il 3 gennaio e il 12 dicembre 1969 sono state censite 145 esplosioni, che, pur non facendo vittime, contribuiscono a creare un clima di paura. Anche se la maggior parte sono attribuibili all'estrema destra, la stampa di orientamento conservatore o moderato - orientata anche da informazioni provenienti dalle questure - addita spesso gli anarchici e l'area dell'estrema sinistra come

\footnotetext{
${ }^{9}$ Ibidem, p. 70. Sull'importanza del convegno dell'Istituto Pollio nella definizione della strategia della tensione e nel coagularsi dei gruppi dell'estrema destra eversiva cfr. FERRARESI, Franco, Minacce alla democrazia. La destra radicale e la strategia della tensione in Italia nel dopoguerra, Milano, Feltrinelli, 1995, pp. 140-144. Sui rapporti tra servizi segreti statunitensi e organizzazioni italiane anticomuniste cfr. PACINI, Giacomo, Le altre Gladio. La lotta segreta anticomunista in Italia 1943-1991, Torino, Einaudi, 2014.

${ }^{10}$ DONDI, Mirco, L'eco del boato, cit., p. 76.

${ }^{11}$ Ibidem, p. 30.
} 
responsabili, «creando il nesso manifestazioni di piazza-attentati per indirizzare l'opinione pubblica su una linea di ritorno all'ordine» ${ }^{12}$. In alcuni casi sono i servizi segreti stessi ad interferire nelle indagini. Significativo è a questo proposito il depistaggio legato alla bomba al rettorato di Padova di cui fa le spese il capo della squadra mobile Pasquale Juliano (che aveva individuato correttamente nella cellula padovana di Ordine Nuovo la matrice dell'attentato), destituito dall'incarico in seguito a pressioni dell'Ufficio affari riservati. La ricostruzione giornalistica della morte dell'agente Antonio Annarumma negli scontri a Milano durante lo sciopero generale del 19 novembre 1969 contribuisce ad aumentare la tensione: le principali testate d'informazione accreditano senza ombra di dubbio la tesi del decesso dell'agente in seguito ad un colpo alla testa subito dai manifestanti.

Nel quarto capitolo, dedicato alla strage di Piazza Fontana, gli aspetti più interessanti riguardano le modalità con le quali la maggior parte dei mass media presenta l'attentato, sostenendo quasi immediatamente la "pista anarchica" (anche attraverso un parallelismo con l'attentato al teatro Diana di Milano del marzo 1921) e la versione ufficiale della morte di Pinelli, demonizzando la figura di Valpreda e conferendo grande risalto al messaggio del Presidente della Repubblica Saragat che dà subito nel suo messaggio una versione "orientata" degli eventi. Fanno eccezione, oltre ai giornali di opposizione, testate come «Il Giorno» e «La Stampa», giornalisti come Giorgio Bocca e, in certa misura, anche un servizio della RAI sui funerali delle vittime della strage curato da Sergio Zavoli (non a caso messo sotto tiro dai vertici dell'azienda). Dondi ricostruisce meticolosamente sia i linguaggi comunicativi dei servizi giornalistici e televisivi, sia la rete di informatori e di pressioni politiche e giudiziarie in cui operano i giornalisti che si occupano dell'evento.

Proprio in reazione a questo battage giornalistico, opera la cosiddetta controinformazione, che, decostruendo la versione ufficiale, dà un contributo fondamentale nello smascherare e quindi anche nel disinnescare la strategia della tensione. Nel quinto capitolo l'autore, dopo una ricostruzione delle novità del contesto politico nel 1970-71 e di alcuni suoi attori (da un lato le formazioni della sinistra extraparlamentare, dall'altro un gruppo come Maggioranza silenziosa, in rapporti di contiguità con l'estrema destra eversiva), si occupa dell'attività del «Bollettino della controinformazione democratica», di riviste come «Re nudo» e della genesi del celebre volume $L a$ strage di Stato, che diventerà un best-seller con mezzo milione di copie vendute ${ }^{13}$. Al di là dei risultati conseguiti, la controinformazione, nelle sue varie accezioni, cerca di operare una sorta di rivoluzione copernicana tra l'organo di informazione e il lettore, rendendo quest'ultimo consapevole dei «meccanismi di manipolazione della notizia smontando titoli, contenuti di articoli o

\footnotetext{
${ }^{12}$ Ibidem, p. 106 (corsivo nel testo).

${ }^{13}$ La strage di Stato: controinchiesta, Roma, Samonà e Savelli, 1970. Sul ruolo della controinformazione cfr. anche GIANNULI, Aldo, Bombe a inchiostro, Milano, BUR, 2008.
} 
informando su quanto accade dietro le quinte delle redazioni» ${ }^{14}$. Ciò non esclude, in alcuni casi, che anche i giornalisti impegnati nella controinformazione facciano ricorso a "soffiate" di esponenti dei servizi segreti, interessati a coprire un loro alleato o a mettere in difficoltà un loro avversario. L'autore non manca di dedicare un paragrafo all'influenza della controinformazione nel teatro (Dario Fo e Franca Rame), nella letteratura (Il contesto di Sciascia), nelle canzoni e nel cinema. Poi passa ad analizzare altri eventi (e la loro riproposizione mediatica) ricollegabili alla strategia della tensione, dall'attentato di Gioia Tauro (luglio 1970) al progetto del golpe Borghese (dicembre 1970), sino all'ordigno che nel maggio 1972 causa la morte di tre carabinieri a Peteano nel Friuli, di cui sono responsabili militanti di Ordine Nuovo.

Già a partire da quest'ultimo attentato, però, il clima inizia a cambiare. La stampa conservatrice strumentalizza di meno il caso, mentre il "Corriere della Sera», sotto la nuova direzione di Piero Ottone, inizia a percorrere una strada più autonoma. Riccardo Lombardi, dirigente della corrente di sinistra del Partito socialista, in una postfazione scritta nel settembre 1972 ad un volume del giornalista dell'«Avanti!» Marco Sassano sul caso Pinelli, afferma che grazie all'«incalzante denuncia» di «giornalisti, uomini politici, magistrati e anche uomini del sistema, ma fedeli alla Repubblica», «l'incrocio tra il disegno eversivo fascista e la congiura all'interno dei poteri dello Stato» (con la complicità o quantomeno la tolleranza di parte dei servizi segreti statunitensi), anche se non può dirsi ancora scongiurato, appare quantomeno in crisi $^{15}$.

Il cambiamento d'indirizzo si evidenzia in seguito all'attentato alla questura di Milano del maggio 1973 che provoca quattro morti. L'attentatore, Gianfranco Bertoli, dichiara di essere un anarchico individualista, anche se in realtà ha un passato di informatore per il SIFAR e il SID ed è in contatto con personalità dell'estrema destra. Eppure, «l'ampio schieramento di stampa confluito sulla linea desiderata dagli strateghi della tensione dopo Piazza Fontana non si ritrova per la strage in questura» ${ }^{16}$. In seguito a questo attentato si ha lo scioglimento di Ordine Nuovo (novembre 1973) e l'inchiesta sulla Rosa dei venti che, per la prima volta, «investe i vertici militari con l'accusa di complotto contro lo Stato ${ }^{17}$. Dondi argomenta che vi è un passaggio dalle stragi di provocazione alle stragi di intimidazione. Se l'obiettivo resta sempre lo stesso - destabilizzare le istituzioni democratiche - ora però non serve più nascondere la matrice "nera" degli attentati. Al contrario, questa va esibita come segnale di avvertimento, nei confronti delle istituzioni e della pubblica opinione.

In questo contesto maturano gli attentati a Brescia (maggio 1974) e al treno Italicus (agosto 1974), di cui si occupa il settimo capitolo. Si tratta di un cambiamento ben avvertito da Pasolini

\footnotetext{
${ }^{14}$ DONDI, Mirco, L'eco del boato, cit., p. 225.

${ }^{15}$ LOMBARDI, Riccardo, Una congiura, in SASSANO, Marco, Pinelli. La finestra chiusa, Venezia, Marsilio, 2009 [Ed. orig. La politica della strage, Padova, Marsilio, 1972], pp. 203-205.

${ }^{16}$ DONDI, Mirco, L'eco del boato, cit., p 320.

${ }^{17}$ Ibidem, p, 329.
} 
che nell'articolo prima citato parla di «due differenti, anzi, opposte, fasi della tensione: una prima fase anticomunista (Milano 1969), e una seconda fase antifascista (Brescia e Bologna 1974)» ${ }^{18}$. Mentre la stampa di destra legge l'attentato di Brescia non più nei termini dell'attacco dei "rossi" contro lo stato, ma come «una strage orchestrata dallo Stato per mutare gli equilibri di potere» ${ }^{19}$, l'unanime condanna del fascismo espressa dai partiti dell'arco costituzionale (e dalla stampa di vario colore che vi si riconosce) è spesso guardata con sospetto negli ambienti della controinformazione. Del resto, dopo il risultato del referendum sul divorzio del 1974 e l'avanzata del PCI nelle elezioni amministrative dell'anno successivo, si preparano nuovi equilibri politici che di lì a poco porteranno ai governi di solidarietà nazionale.

Il 1974 è quindi scelto da Dondi come il termine ad quem della narrazione.

Le forzature stragiste del '74, pensate come sbocco a un colpo di Stato, hanno finito per provocare l'abbandono delle strutture del golpe (che nel caso della P2 sono riconvertite), hanno stimolato la funzione investigativa del giornalismo, hanno mostrato ai politici al governo l'impopolarità di posizioni ambigue di fronte allo stragismo nero ${ }^{20}$.

Le trame eversive continueranno, ma dovranno sfruttare altri metodi. È il caso della P2 e del suo Piano di rinascita democratica che segna, come già notava Francesco Biscione, il superamento della strategia della tensione almeno rispetto a come veniva precedentemente intesa ${ }^{21}$. Le attività della commissione d'inchiesta sulle cause della mancata individuazione dei responsabili delle stragi varata dal Parlamento nel 1988 e le inchieste degli anni Novanta (in particolare quella del giudice Salvini) contribuiranno a fare luce ma non dissiperanno mai del tutto quel velo di opacità che ancora avvolge la strategia della tensione. L'opera dello storico risulta fondamentale ma avverte l'autore - può colmare solo in parte «le falle dell'omertà politica e dell'evasione giudiziaria» ${ }^{22}$.

\footnotetext{
${ }^{18}$ PASOLINI, Pier Paolo, Scritti corsari, cit., p. 88.

${ }^{19}$ DONDI, L'eco del boato, cit., p. 358.

${ }^{20}$ Ibidem, p. 385.

${ }^{21}$ BISCIONE, Francesco M., Il sommerso della Repubblica. La democrazia italiana e la crisi dell'antifascismo, Torino, Bollati Boringhieri, 2003, p. 118.

${ }^{22}$ DONDI, L'eco del boato, cit., p. 404.
} 


\section{L'AUTORE}

Luca BUFARALE ha conseguito nel 2008 la Laurea Specialistica in Storia d'Europa (indirizzo contemporaneo) presso l'Università di Bologna e nel 2012 il Dottorato di Ricerca in Scienze storiche presso l'Università di Padova. É autore del volume Riccardo Lombardi. La giovinezza politica 1919-1949 (Roma, Viella, 2014). Ha pubblicato saggi e recensioni in «Studi storici», «Il Mestiere di storico», «Storicamente», «Annali della Fondazione Ugo La Malfa», «Quaderni della Fondazione Giacomo Brodolini», «Il Ponte» e in diversi volumi collettanei. Fa parte della redazione della rivista «Diacronie. Studi di storia contemporanea».

URL: < http://www.studistorici.com/progett/autori/\#Bufarale $>$ 